\title{
Increased fruit selection and consumption following an appearance-based vs a health-based health promotion poster
}

\author{
K. M. Appleton \\ Bournemouth University, Poole House, Fern Barrow, Poole, Dorset, BH12 5BB, UK
}

Health promotion posters to improve health behaviours typically use long term health or severe illness as primary motivators ${ }^{(1)}$. Recent research in various fields however, suggests success for promoting health behaviours using appearance based motivators. Reading an essay on the effects of sun on appearance was more effective than reading an essay on the health risks of excessive sun exposure and reading a control essay for safe sun behaviours ${ }^{(2)}$, UV photographs resulted in less skin darkening at $4-5$ month follow-up, and photoaging information resulted in more sun protective behaviours and less skin darkening at 4-5 month and 12 month follow-up ${ }^{(3)}$, and messages emphasizing the disfiguring aspects of osteoporosis increased intentions to perform protective behaviours compared to controls ${ }^{(4)}$. Limited research however has been conducted in the area of healthy eating. This study investigated the impact of an appearance-based and a health-based health promotion poster on intentions to consume fruit, immediate fruit selection and subsequent fruit consumption.

One-hundred and sixty-six participants who were either primarily health motivated $(\mathrm{N}=59)$, primarily appearance motivated $(\mathrm{N}=$ $71)$, or equally health and appearance motivated $(\mathrm{N}=36)$ viewed either a health-based $(\mathrm{N}=84)$ or an appearance-based health promotion poster $(\mathrm{N}=82)$. Intentions to consume fruit the following day were subsequently recorded by questionnaire, selection of fruit from a range of snacks was recorded as a measure of immediate consumption, and subsequent consumption was requested the following day by email. Background details on usual fruit consumption, liking for fruit, and motivation were assessed prior to viewing the poster.

No effects were found on intentions to consume fruit (largest $\mathrm{F}(1,160)=0 \cdot 20, p=0 \cdot 66$ ). Compared to the health based poster however, the appearance-based poster resulted in increased fruit selection at the end of the study $(\mathrm{F}(1,148)=7 \cdot 66, p<0 \cdot 01)$ and increased reporting of subsequent fruit consumption $(\mathrm{F}(1,88)=5 \cdot 11, p=0 \cdot 03)$. No differences were found dependent on whether individuals reported more usual motivation by health, appearance or neither (largest $\mathrm{F}(2,148)=0 \cdot 50, p=0 \cdot 61$ ).

These findings suggest that appearance-based health promotion posters can increase fruit selection and consumption more effectively than health-based posters. Research on other health behaviours also suggests improved health behaviours following appearance-based compared to health-based messages ${ }^{(2,3,4)}$, but the reasons for effects are unclear. Effects here were found in behaviour as opposed to intentions, and regardless of individual motivation. These findings may suggest an effect due to immediate cues, as opposed to careful reasoning, but more work is clearly needed here. The use of appearance-based interventions for improving healthy eating, however, deserves further investigation.

1. Ashfield-Watt PAL (2006) Fruit and vegetables, 5+ a day: Are we getting the message across? Asia Pacific J ClinNutr 15, 245-252.

2. Jones JL, Leary MR (1994) The effect of appearance-based admonitions against sun exposure on tanning intentions in young adults. Health Psych 13, 86-90.

3. Mahler HI, Kulik JA, Gerrard M, Gibbons FX (2007) Long-term effects of appearance-based interventions on sun protection behaviors. Health Psych 26, 350-60

4. Smith Klohn L, Rogers RW (1991) Dimensions of the severity of a health threat. Health Psych 10, 323-329. 